\title{
Schematic Coupled-Channel Calculations for Nuclear Reactions with Unstable Beams
}

\author{
W.H.Z. Cárdenas, \\ Instituto de Física, Universidade de São Paulo, C.P. 66318, 05389-970, São Paulo, Brazil \\ L.F. Canto, \\ Instituto de Física, Universidade Federal do Rio de Janeiro, C.P. 68528, 21941-972 Rio de Janeiro, Brazil \\ R. Donangelo, \\ Instituto de Física, Universidade Federal do Rio de Janeiro, C.P. 68528, 21941-972 Rio de Janeiro, Brazil \\ N. Carlin, and M.S. Hussein \\ Instituto de Física, Universidade de São Paulo, C.P. 66318, 05389-970 São Paulo, Brazil
}

Received on 12 March, 2004

\begin{abstract}
We study nuclear reactions in collisions of unstable projectiles with heavy targets. For this purpose, we use a simple approximation for the breakup channel and treat two-neutron halos as a single particle. We then evaluate cross sections for collisions of ${ }^{6} \mathrm{He}$ projectiles with targets of ${ }^{238} \mathrm{U}$ and ${ }^{209} \mathrm{Bi}$. Comparing our results with recently measured fusion cross sections, we conclude that the large enhancement observed in ${ }^{6} \mathrm{He}+{ }^{238} \mathrm{U}$ fusion at sub-barrier energies cannot be explained by the coupling with the breakup channel. The effects of the halo on other reaction channels are also investigated. Coulomb-nuclear interference in the breakup channel is discussed.
\end{abstract}

\section{Introduction}

The influence of channel-coupling on fusion reactions has been studied by several authors [1, 2, 3]. In collisions of stable nuclei, the main conclusion of these studies is that the coupling leads to a strong enhancement of the fusion cross section at sub-barrier energies. The discovery that some neutron-rich unstable nuclei, such as ${ }^{11} \mathrm{Li}$ or ${ }^{6} \mathrm{He}$, exhibit a neutron halo renewed the interest in this theme [4]. The presence of a halo in the nuclear density contributes to the reduction of the Coulomb barrier and, therefore, to an increase of the fusion cross section. This is an effect of static nature. On the other hand, there are important dynamic effects. The weak binding of the halo neutrons leads to a strong coupling between the elastic and breakup channels, which strongly affects the fusion process. In the first place, the concept of fusion becomes ambiguous. There is the possibility of fusion through the formation of a compound nucleus containing all the projectile's and target's nucleons, like in collisions of strongly bound nuclei. This process is called complete fusion and we denote the corresponding cross section by $\sigma_{C F}$. However, there is also the possibility that fusion follows the breakup process. In this case, one or more of the fragments may emerge from the interaction region, with the compound nucleus being formed by the target and the remaining projectile's fragments. This process is named incomplete fusion and we denote the cross section associated to it by $\sigma_{I C F}$. For instance, in the ${ }^{6} \mathrm{He}+{ }^{238} \mathrm{U}$ collision the fragmentation of the ${ }^{6} \mathrm{He}\left({ }^{6} \mathrm{He} \longrightarrow 2 \mathrm{n}+{ }^{4} \mathrm{He}\right)$ can be followed by the incomplete fusion reaction ${ }^{4} \mathrm{He}+{ }^{238} \mathrm{U} \longrightarrow{ }^{242} \mathrm{Pu}$.

The first theoretical works in the area of fusion with unstable nuclei $[5,6,7,8]$ have appeared in the beginning of the last decade, for collisions involving ${ }^{11} \mathrm{Li}$. These works were based on schematic models that focused different aspects of the problem. For this reason, they led to conflicting conclusions. Hussein et al. [6] and Takigawa et al. [7] took into account the effects of the breakup on the elastic channel through a polarization potential. These authors made the hypothesis that the breakup was a direct (not a resonant) process and that this channel would only contribute to incomplete fusion. Its contribution to the complete fusion cross section would come from a sequential process of higher order and for this reason it could be neglected. In this way, $\sigma_{C F}$ would be determined exclusively by the elastic wave function. Compared with the fusion cross section in the absence of channel-coupling, the results of this model showed a strong reduction above and in the neighborhood of the Coulomb barrier and a pronounced enhancement at energies much below the barrier. If the polarization poten- 
tial had been calculated in an exact way, the results obtained with this model would be identical to those of the coupledchannels method. However, their polarization potential [11] contained several approximations. Among them was the neglect of the real part of the potential, which produces a reduction of the fusion barrier and, consequently, an increase of the cross section at sub-barrier energies. Although this effect has been taken into account in an approximate way, the adopted procedure is unsatisfactory at energies close to the Coulomb barrier. The model of Dasso and Vitturi [8] is of a diametrically opposite nature. These authors treated the breakup channel as a bound channel and calculated the fusion cross section adding contributions from all channels. If the breakup process is direct and the sequential complete fusion can be neglected, as assumed in $[6,9,7]$, the cross section of [8] corresponds to the total fusion $\sigma_{T F}=\sigma_{C F}+\sigma_{I C F}$ and, therefore, should not be compared with $\sigma_{C F}$. On the other hand, if the breakup occurs through a resonance of much larger half-life than the collision time, incomplete fusion does not exist. In this case, $\sigma_{T F}=\sigma_{C F}$ and the comparison would be appropriate. Some of these questions found answers in the recent calculations by Hagino et al. [12] and Diaz-Torres et al. [13, 14]. These calculations use the coupled-channels method, approximating the breakup channel by a finite set of states in the continuum. This procedure is known as Continuum Discretized coupled-channels (CDCC). Its implementation has as starting point the FRESCO code [15] and the continuum discretization method described in [16]. There are two differences between the calculations of refs. [12] and [13]. The first one is that Diaz-Torres and Thompson use a wider continuum discretization mesh, reaching higher energies. The second is that these authors take into account the coupling among continuum states, which has been neglected in [12]. In this way, the results of ref.[13] are the most accurate presently available. They lead to two important conclusions. The first is that in collisions with heavy targets the coupling with the breakup channel leads to a substantial increase of the complete fusion cross section at sub-barrier energies and to a strong suppression above the barrier. The second conclusion is that the results are very sensitive to the set of states used to describe the continuum and also to the continuum-continuum coupling. It is important to observe, however, that the CDCC method still presents limitations. It has not been implemented in the case of halos with more than one nucleon. Therefore, it cannot be applied to important cases such as fusion reactions induced by ${ }^{11} \mathrm{Li}$ and ${ }^{6} \mathrm{He}$ beams.

Several experimental studies of reactions with unstable beams have been carried out in the last few years. Fusion cross sections have been measured in experiments with beams of ${ }^{11} \mathrm{Be}[17,18,19,20],{ }^{6} \mathrm{He}[21,22],{ }^{17} \mathrm{~F}[24]$ and ${ }^{28} \mathrm{~S}$ [25]. The experimental separation of $\sigma_{C F}$ and $\sigma_{I C F}$ presents difficulties, specially when uncharged fragments are produced in the breakup of the projectile. For this reason, most of the experimental works ignore the distinction between $\sigma_{C F}$ and $\sigma_{I C F}$ and measure the total fusion cross section, $\sigma_{T F}=\sigma_{C F}+\sigma_{I C F}$. Some authors use a different concept of complete fusion, defining it as the process where the compound nucleus contains the total charge of projectile and target. In some cases, as in the fusion after the breakup ${ }^{6} \mathrm{Li} \longrightarrow{ }^{4} \mathrm{He}+\mathrm{d}$, the two definitions are equivalent. However, after breakups like ${ }^{6} \mathrm{He} \longrightarrow{ }^{4} \mathrm{He}+2 \mathrm{n}$ or ${ }^{11} \mathrm{Li} \longrightarrow{ }^{9} \mathrm{Li}+2 \mathrm{n}$ the two definitions are different. Therefore, for comparisons between experimental data and theoretical predictions it is important to make sure that corresponding cross sections are being taken. A good summary of the current experimental situation is presented by Alamanos et al. [23]. The fusion cross sections for collisions of ${ }^{6} \mathrm{He}$ and ${ }^{11} \mathrm{Be}$ are compared with results for the corresponding stable isotopes and the same targets. This comparison leads to two conclusions. The first is that the cross sections for the unstable projectiles present pronounced differences with respect to the results for the corresponding stable isotopes. The second is that it is not possible to establish a pattern for these differences. In the ${ }^{6} \mathrm{He}+{ }^{238} \mathrm{U}$ collision at sub-barrier energies the observed cross section is dramatically larger than that for ${ }^{4} \mathrm{He}+{ }^{238} \mathrm{U}$ [22]. At energies above the barrier the cross section for the ${ }^{6} \mathrm{He}+{ }^{238} \mathrm{U}$ system remains higher but the difference is not so large. In collisions of the same projectiles on a ${ }^{209} \mathrm{Bi}$ target [21] a similar behavior is observed below the barrier. However, the ${ }^{6} \mathrm{He}$ and ${ }^{4} \mathrm{He}$ cross sections are approximately the same at energies above the barrier. For ${ }^{9,11} \mathrm{Be}$ beams and ${ }^{209} \mathrm{Bi}$ targets the situation is very different [19]. Above the Coulomb barrier, the cross section for the unstable isotope ${ }^{11} \mathrm{Be}$ is larger than that for the stable one, ${ }^{9} \mathrm{Be}$. On the other hand, these cross sections are very similar at energies below the barrier. The total fusion cross sections measured in the ${ }^{38} \mathrm{~S}+{ }^{181} \mathrm{Ta}$ collisions [25] (neutron-rich projectile) and ${ }^{17} \mathrm{~F}+{ }^{208} \mathrm{~Pb}$ [24] (proton-rich projectile) did not show significant differences when compared with results obtained for stable projectiles.

From the above discussion, it is clear that further theoretical and experimental studies are needed for an appropriate understanding of the nuclear reactions induced by unstable beams. In the present paper we use a simple approximation for the breakup channel proposed in a previous paper [26] to carry out coupled-channel calculations for fusion, breakup, reaction and elastic cross sections. In particular, we calculate the fusion cross sections for the ${ }^{4,6} \mathrm{He}+{ }^{238} \mathrm{U}$ and ${ }^{4,6} \mathrm{He}+{ }^{209} \mathrm{Bi}$ systems, which have recently been measured $[22,21]$. The rest of this paper is organized as follows. In section 2 we derive optical potentials by the folding method and present the details of our coupled-channel calculations. In section 3 we perform numerical calculations for the ${ }^{6} \mathrm{He}+{ }^{238} \mathrm{U}$ and ${ }^{6} \mathrm{He}+{ }^{209} \mathrm{Bi}$ systems and discuss the importance of the static and the dynamic effects of the ${ }^{6} \mathrm{He}$ halo. Finally, in section 4 we present the conclusions of this work.

\section{Coupled-channel calculations in the schematic model}

Our calculations for the ${ }^{6} \mathrm{He}+{ }^{238} \mathrm{U}$ and ${ }^{6} \mathrm{He}+{ }^{238} \mathrm{U}$ systems follow three steps: (a) calculation of optical potentials, (b) choice of the intrinsic states for the coupled-channel equations and (c) calculation of coupling matrix-elements and 
solution of the coupled radial equations.

\subsection{The optical potential}

The optical potential for a nuclear collision can be determined with the help of some theoretical model or by a phenomenological procedure. In the present work we use the single folding method [27], where the real part of the optical potential is given by the integral

$$
V_{N}(\mathbf{r})=\int v_{n-A_{T}}\left(\mathbf{r}-\mathbf{r}^{\prime}\right) \rho\left(\mathbf{r}^{\prime}\right) d^{3} \mathbf{r}^{\prime}
$$

$$
V_{C}(r)=\left\{\begin{array}{l}
\frac{Z_{1} Z_{2} e^{2}}{2 R_{C}}\left(3-\frac{r^{2}}{R_{C}^{2}}\right), \\
\frac{Z_{1} Z_{2} e^{2}}{r}, \quad r \geq R_{C}
\end{array}\right.
$$

and $W(r)$ is the absolute value of a strongly absorptive imaginary potential with small radius and diffusivity. We use a Woods-Saxon parametrization with $W_{0}=50 \mathrm{MeV}$, $r_{i}=1.0 \mathrm{fm}$ and $a_{i}=0.10 \mathrm{fm}$.

In the calculation of the folding potential we use the nucleon-target interaction of Madland and Young [28] (dropping the spin-orbit part),

$$
v_{n-A_{T}}\left(\mathbf{r}-\mathbf{r}^{\prime}\right)=-V_{0} f_{r}(x),
$$

where $x=\left|\mathbf{r}-\mathbf{r}^{\prime}\right|$,

$$
V_{0}=\left[50.378-27.073\left(\frac{N-Z}{A}\right)-0.354 E_{L a b}\right]
$$

and

$$
f_{r}(x)=\frac{1}{1+\exp \left[\left(x-R_{r}\right) / a_{r}\right]} .
$$

The interaction parameters are

$$
R_{r}=1.264 A_{T}^{1 / 3} \mathrm{fm} \quad \text { e } a_{r}=0.612 \mathrm{fm} .
$$

However, as we will discuss below, we leave room for slight adjustments of these parameters in order do get a good description of the fusion cross sections for collisions of the stable isotope ${ }^{4} \mathrm{He}$ with the ${ }^{238} \mathrm{U}$ and ${ }^{209} \mathrm{Bi}$ targets.

For ${ }^{4} \mathrm{He}$ projectiles, we use the Gaussian form factor

$$
\rho(r)=C \exp \left(-r^{2} / \gamma^{2}\right)
$$

The parameters $C$ and $\gamma$ are obtained by the condition that the norm and the r.m.s radius have the correct values. That is

$$
\int \rho(r) d^{3} r=\mathcal{N} ; \quad \frac{1}{\mathcal{N}} \int r^{2} \rho(r) d^{3} r=r_{r m s}^{2}
$$

Above, $v_{n-A_{T}}$ is an appropriate nucleon-target interaction and $\rho\left(\mathbf{r}^{\prime}\right)$ is the projectile's density. Firstly, we look for a nucleon-target interaction that leads to a good description of the reactions with the stable ${ }^{4} \mathrm{He}$ projectiles. The static effects of the halo are then introduced into the optical potential through the folding of this interaction with a realistic density for ${ }^{6} \mathrm{He}$.

The full optical potential is

$$
U(r)=V_{N}(r)+V_{C}(r)-i W(r),
$$

where $V_{C}(r)$ is the Coulomb potential

$$
r \leq R_{C}=1.2\left(A_{1}^{1 / 3}+A_{2}^{1 / 3}\right)
$$

In the present case we set $\mathcal{N}=4$ and $r_{r m s}=1.49 \mathrm{fm}$ [29].
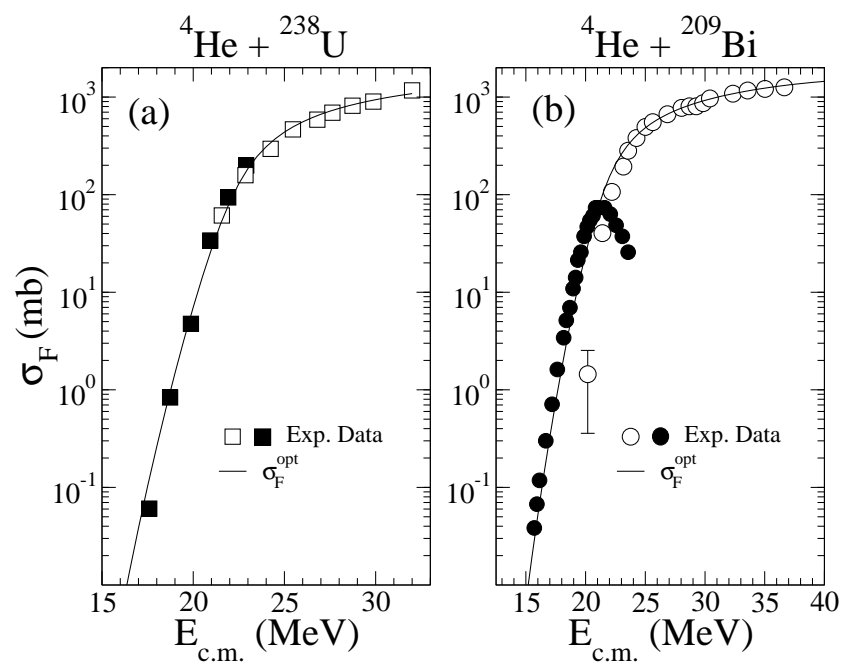

Figure 1. Fusion cross sections in collisions of ${ }^{4} \mathrm{He}$ with (a) ${ }^{238} \mathrm{U}$ and (b) ${ }^{209} \mathrm{Bi}$. The solid lines represent calculations with the optical potentials given by the folding model. The squares and circles are the experimental data of [34] (open squares), [22] (solid squares), [35] (open circles) and [36] (solid circles).

In Fig. 1 the calculated fusion cross sections for the ${ }^{4} \mathrm{He}+{ }^{238} \mathrm{U}$ (Fig. 1(a)) and ${ }^{4} \mathrm{He}+{ }^{209} \mathrm{Bi}$ (Fig. 1(b)) collisions are compared with the experimental values. In both cases the folding potentials lead to very accurate description of the data. For the ${ }^{4} \mathrm{He}+{ }^{238} \mathrm{U}$ collision, the calculations were performed without any modification of the original parameters of the Madland and Young interaction. For the ${ }^{209} \mathrm{Bi}$ the radius parameter was slightly modified, as indicated below

$$
R_{r}=1.264 A_{T}^{1 / 3} \mathrm{fm} \longrightarrow R_{r}=1.220 A_{T}^{1 / 3} \mathrm{fm} .
$$


We now consider the collisions of ${ }^{6} \mathrm{He}$ projectiles with the same targets. If all the ${ }^{6} \mathrm{He}$ nucleons where strongly bound, like in ${ }^{4} \mathrm{He}$, the density would be given by a Gaussian parametrization with new values of the constants $C$ and $\gamma$. They would be given by eq.(8) with

$\mathcal{N}=6 ; r_{r m s}\left({ }^{6} \mathrm{He}\right)=r_{r m s}\left({ }^{4} \mathrm{He}\right) \times\left(\frac{6}{4}\right)^{1 / 3}=1.71 \mathrm{fm}$.
However, the Gaussian shape is not appropriate for a halo nucleus. One should use instead a realistic parametrization, consistent with the halo of ${ }^{6} \mathrm{He}$ [29]. This parametrization is based on the symmetrized Fermi distribution of [30],

$$
\rho_{S F}(r)=\rho_{0}\left[\left(1+\exp \left(\frac{r-R}{a}\right)\right)^{-1}+\left(1+\exp \left(\frac{-r-R}{a}\right)\right)^{-1}-1\right]
$$

with

$$
\rho_{0}=\frac{3 A}{4 \pi R^{3}}\left[1-\left(\frac{\pi a}{R}\right)^{2}\right]^{-1},
$$

$A=6, R=1.23 A^{1 / 3} \mathrm{fm}$ and $a=0.57 \mathrm{fm}$. This parametrization leads to the r.m.s. radius $r_{r m s}=2.30 \mathrm{fm}$, which is appreciably larger than the one in eq.(9), obtained without the halo. In this way, one obtains a lower barrier as shown in table I.

\subsection{The channel space in the schematic model}

Including the breakup channel in coupled-channel calculations is a very hard task. The difficulty arises from the fact that the breakup channel is represented by an infinite set of three or more particles in the continuum. For practical purposes it is necessary to represent the continuum by a finite set of states. In the case of one neutron halos, this can be done by the CDCC method [12, 13, 14]. However, the implementation of this procedure is very complicated. In the present work we use a qualitative model based on two approximations. The first one is to replace the two neutrons of the ${ }^{6} \mathrm{He}$ halo by a single particle, the di-neutron. This approximation, which lead to reasonable results in the breakup of ${ }^{11} \mathrm{Li}$ [31], amounts to neglecting the relative motion between the two neutrons. The second approximation consists of replacing the infinite set of breakup states by a single channel, which represents them in an effective way [32]. Since the coupling is dominated by the electric dipole term, and the ${ }^{6} \mathrm{He}$ ground state has spin zero, we assume that the effective channel has spin 1 . We neglect the relative energy of the fragments in the breakup channel and adopt for the effective channel the breakup threshold energy, $\epsilon_{1}=0.975$ $\mathrm{MeV}$.

The approximation of the continuum by a single effective channel would be fully justified in the case of breakup through a sharp resonance, with a lifetime much longer than the collision time. However, for direct breakup, this approximation would not be justified and it would lead to wrong predictions for $\sigma_{C F}$. In this case, the effective channel would dominantly contribute to the incomplete fusion cross section. An approximate way to take this into account is to use the effective channel approximation but neglecting its contribution to $\sigma_{C F}$. Using the notation $\sigma_{F}^{(\alpha)}$ for the contribution from channel- $\alpha$ to the fusion cross section, this procedure yields

$$
\sigma_{C F} \simeq \sigma_{F}^{(0)}
$$

On the other hand, the consequences of this approximation on $\sigma_{T F}$ should be less important since the contributions from all channels are summed, independently of the reaction mechanism. This is the cross section that will be compared with the experimental data of [22] and [21].

\subsection{The radial equations and the coupling matrix-elements}

The coupled radial equations are

$$
\begin{aligned}
{\left[E_{\alpha}+\frac{\hbar^{2}}{2 \mu}\left(\frac{d^{2}}{d r^{2}}-\frac{l(l+1)}{r^{2}}\right)\right.} & -\mathrm{U}(\mathrm{r})] u_{\alpha l, \alpha_{0} l_{0}}^{J}\left(k_{\alpha}, r\right) \\
& =\sum_{\alpha^{\prime} l^{\prime}} \mathbb{U}_{\alpha l, \alpha^{\prime} l^{\prime}}^{J}(r) u_{\alpha^{\prime} l^{\prime}, \alpha_{0} l_{0}}^{J}\left(k_{\alpha^{\prime}}, r\right),
\end{aligned}
$$


where $\alpha$ takes the values 0 (elastic channel) and 1 (effective breakup channel). We use the same optical potential of the previous sections for both channels.

The large values of the breakup cross section in collisions of halo nuclei with heavy targets indicate that the long range electric dipole coupling dominates. In our calculations we take into account both the Coulomb and the nuclear couplings and approximate them by their dipole terms. The electric dipole matrix elements are given by [27]

$$
\mathbb{U}_{1 l, 0 l_{0}}^{J}(r)=(-)^{J+1} i^{l-l_{0}} \hat{l} \hat{l}_{0} \sqrt{\frac{4 \pi}{3}} F_{C}(r)\left(\begin{array}{ccc}
l & 1 & l_{0} \\
0 & 0 & 0
\end{array}\right)\left\{\begin{array}{ccc}
J & 1 & l \\
1 & l_{0} & 0
\end{array}\right\}
$$

with the notation $\hat{l}=\sqrt{2 l+1}\left(\hat{l}_{0}=\sqrt{2 l_{0}+1}\right)$. The electric dipole form factor is approximated as

$$
F_{C}(r)=\left\{\begin{array}{cc}
A \frac{r}{R_{C}^{3}}\left(4-\frac{3 r}{R_{C}}\right), & r<R_{C} \\
A \frac{1}{r^{2}}, & r \geq R_{C} .
\end{array}\right.
$$

Above, $\left(\begin{array}{ccc}l & 1 & l_{0} \\ 0 & 0 & 0\end{array}\right)$ and $\left\{\begin{array}{ccc}J & 1 & l \\ 1 & l_{0} & 0\end{array}\right\}$ are the usual 3J and $6 \mathrm{~J}$ symbols [33] and the strength $A$ is

$$
A=e Z_{T} \sqrt{B(E 1,0 \rightarrow 1)} .
$$

In this way, the matrix-elements $\mathbb{U}_{1 l, 0 l_{0}}^{J}(r)$ are completely determined in terms of the reduced transition probability $B(E 1,0 \rightarrow 1)$. We adopt the $B(E 1,0 \rightarrow 1)$ value of the cluster model,

$$
B(E 1,0 \rightarrow 1)=\frac{3 \hbar e^{2}}{16 \pi \epsilon_{1} \mu_{2 n-{ }^{4} H e}}=1.37 \mathrm{e}^{2} \mathrm{fm}^{2} .
$$

Above, $\epsilon_{1}$ is the breakup threshold for ${ }^{6} \mathrm{He}$ and $\mu_{2 n-{ }^{4} \mathrm{He}}$ is the reduced mass for the relative motion between the dineutron and the ${ }^{4} \mathrm{He}$-core.

Recently, Hagino et al. [12] pointed out that the nuclear coupling to the breakup channel plays a very important role in the fusion of weakly bound nuclei. However, the inclusion of the corresponding nuclear matrix-elements in our schematic model presents some difficulties. Since we neglect the kinetic energy of the fragment-fragment motion, the normalization factor of the effective channel goes to zero. Therefore, the matrix-elements between the elastic and the effective channel vanish. To avoid this problem we follow the procedure introduced in ref. [26], which is described below. The nuclear part of the coupling interaction, $\mathcal{V}$, is obtained by summing the interaction between the ${ }^{4} \mathrm{He}$ fragment and the target $\left(V^{4} \mathrm{He}\right)$ with that between the dineutron and the target $\left(V_{2 n}\right)$ and subtracting the projectiletarget potential, $V_{N}(\mathbf{r})$. That is

$$
\mathcal{V}(\mathbf{r}, \mathbf{x})=V_{{ }^{4} \mathrm{He}}(\mathbf{r}-\mathbf{x} / 3)+V_{2 n}(\mathbf{r}-2 \mathbf{x} / 3)-V_{N}(\mathbf{r}),
$$

where $\mathbf{x}$ is the vector going from the di-neutron to the ${ }^{4} \mathrm{He}$ core. The potential $V_{2 n}$ is the nucleon-target interaction multiplied by two while $V_{4} \mathrm{He}$ and $V_{N}(\mathbf{r}) \equiv V_{6} \mathrm{He}$ are respectively the folding potentials for ${ }^{4} \mathrm{He}$ and ${ }^{6} \mathrm{He}$, discussed in the previous sub-section. Carrying out partial-wave expansions and keeping only the dipole term (as we did for the Coulomb coupling), we get

$$
\mathcal{V}(\mathbf{r}, \mathbf{x})=\sum_{\mu} Y_{1 \mu}(\hat{\mathbf{r}}) Y_{1 \mu}^{*}(\hat{\mathbf{x}}) \mathcal{V}_{1}(r, x) .
$$

The matrix-element between the projectile states $\phi_{i}(\mathbf{x})$ and $\phi_{j}(\mathbf{x})$ may be calculated with the help of Wigner-Eckart's theorem. We are interested in the case where one of these states is the ground state, with angular momentum 0 and intrinsic wave function $u_{g s}(x)$. The other is some state in the continuum with angular momentum 1 , energy $\varepsilon$ and radial wave function $u_{\varepsilon}(x)$. The desired matrix-element is given in terms of the nuclear form factor

$$
F_{N}(\varepsilon, r)=\int_{0}^{\infty} d r u_{g s}(x) \mathcal{V}_{1}(r, x) u_{\varepsilon}(x)
$$

The radial wave functions $u_{g s}$ and $u_{\varepsilon}$ are solutions of the radial equation associated with the absolute value of the vector $\mathrm{x}$. The depth of the potential well giving the interaction between the fragments is determined by the condition that its second S-state (the first is excluded by Pauli's principle) has binding energy $-0.975 \mathrm{MeV}$. Owing to the normalization of $u_{\varepsilon}(x)$, the form factor goes to zero in the limit $\varepsilon \rightarrow 0$, which was assumed for the effective breakup channel. To avoid this difficulty, we adopt the radial dependence obtained in eq.(19) but treat its normalization as a free parameter. That is

$$
F_{N}(r)=\mathcal{F}_{0} g(r)
$$

with

$$
g(r)=\lim _{\varepsilon \rightarrow 0}\left[\frac{F_{N}(\varepsilon, r)}{F_{N}(\varepsilon, 0)}\right] .
$$

To estimate the strength $\mathcal{F}_{0}$, we adopt the following procedure. Firstly, we evaluate the electric dipole form factor using the analog of eq.(19) for the Coulomb interaction. The 
resulting Coulomb form factor is then compared with the nuclear one. For the ${ }^{6} \mathrm{He}+{ }^{238} \mathrm{U}$ system, the two form factors have the same strength at $r \simeq 16 \mathrm{fm}$. In our coupledchannel calculations we use the Coulomb form factor given by eqs.(14) to (16) and choose $\mathcal{F}_{0}$ by imposing that the nuclear and the Coulomb form factors have the same value at $r \simeq 16 \mathrm{fm}$. Further details of this procedure are given in ref. [26]. Since the nuclear form factor for the ${ }^{6} \mathrm{He}+{ }^{209} \mathrm{Bi}$ system should be very similar to that for ${ }^{6} \mathrm{He}+{ }^{238} \mathrm{U}$, we use the same nuclear form factors in both calculations.

Solving the coupled-channel equations, we evaluate the scattering amplitudes $\bar{f}_{\alpha}(\theta)$, for $\alpha=0$ and 1 . The elastic and the breakup cross sections are then given by

$$
\begin{aligned}
\frac{d \sigma_{e l}(\theta)}{d \Omega} & =\left|f_{C}(\theta)+\bar{f}_{0}(\theta)\right|^{2} \\
\frac{d \sigma_{B u p}(\theta)}{d \Omega} & =\frac{k_{1}}{k_{0}}\left|\bar{f}_{1}(\theta)\right|^{2} .
\end{aligned}
$$

The total fusion cross section can be written

$$
\sigma_{T F}=\sigma_{F}^{(0)}+\sigma_{F}^{(1)}
$$

with $^{1}$

$$
\sigma_{F}^{(\alpha)}=(2 \pi)^{3} \frac{k_{0}}{E}\left\langle\psi_{\alpha}^{(+)}\left|W_{\alpha}\right| \psi_{\alpha}^{(+)}\right\rangle
$$

for $\alpha=0,1$.

\section{Application: study of the ${ }^{6} \mathrm{He}+{ }^{238} \mathrm{U}$ and ${ }^{6} \mathrm{He}+{ }^{209} \mathrm{Bi}$ collisions}

\subsection{Fusion cross sections}

In Fig. 2, we present fusion cross sections for collision of ${ }^{6} \mathrm{He}$ projectiles with ${ }^{238} \mathrm{U}$ (a) and ${ }^{209} \mathrm{Bi}$ (b) targets. The lines were obtained with our schematic model with different approximations. These results are compared with the data of Trotta et al.[22] (squares) and Kolata et al.[21] (circles). The dotted and the dashed lines are results of optical model calculations, without channel-coupling. In the former, the optical potential is calculated by the folding method with a density without the halo. In the latter, the density includes the ${ }^{6} \mathrm{He}$ halo. The dot-dashed and the solid lines are results of coupled-channel calculations with the optical potential containing the static effects of the halo. In the former the coupling is purely Coulomb while the latter includes both the Coulomb and the nuclear couplings, as discussed in the previous section.
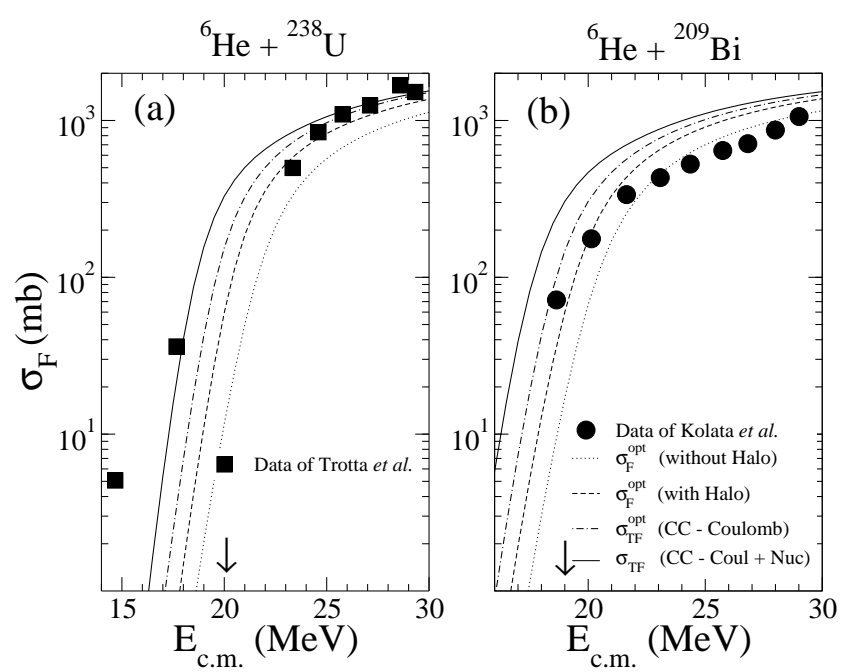

Figure 2. Experimental and theoretical fusion cross sections in the collisions of ${ }^{6} \mathrm{He}$ projectiles with (a) ${ }^{238} \mathrm{U}$ and (b) ${ }^{209} \mathrm{Bi}$. The data are from [22] (squares) and [21] (circles). The results obtained without channel-coupling are represented by dotted (optical potential without halo) and dashed (optical potential with halo) lines. The results from coupled-channel calculations are represented by dot-dashed (Coulomb coupling) and solid (Coulomb plus nuclear coupling) lines.

The trends of the calculations for the two targets (figures 2(a) and 2(b)) are basically the same. Comparing the optical model calculations without (dotted line) and with (dashed lines) the static effects of the halo, we conclude that the halo produces large enhancements of the fusion cross sections above and below the Coulomb barrier. The dynamic effects arising from channel-coupling lead to further enhancements, specially at sub-barrier energies.

Comparing the calculations with the experimental data, we reach different conclusions for the ${ }^{238} \mathrm{U}$ and the ${ }^{209} \mathrm{Bi}$ targets. In the case of ${ }^{238} \mathrm{U}$, the optical model calculation without effects of the halo (dotted line) yields results much below the data, in the whole energy range. The three remaining calculations are much closer to the experiment at above barrier energies. However, none of these calculations provides a good description of the data at sub-barrier energies. The result of the coupled-channel calculation with the Coulomb plus nuclear couplings is close to the sub-barrier data point at $\sim 17 \mathrm{MeV}$ but it is much lower than the one at $\sim 15 \mathrm{MeV}$. Since our model tends to overestimate the fusion cross section, this result suggests that the large enhancement of the experimental data at these energies cannot arise from the ${ }^{6} \mathrm{He}$ halo. We should also call attention to the possibility of these data being inaccurate, owing the experimental technique used in the experiment of ref.[22]. Since fission is the only relevant channel in the decay of the coupound nucleus, the fusion cross section was measured through the detection of fission events. However, the 1 n- and 2 n-transfer channels also lead to fission. Trotta $e t$ al. tried to eliminate the contributions from these processes through an anti-coincidence with the alpha-particle produced in the transfer processes. However, some residual contributions from these processes, which would be relevant at very low energies, may have been left out. In this way, the high cross sections observed at

\footnotetext{
${ }^{1}$ We assume that $\psi_{\alpha}^{(+)}$is defined with the normalization factor $A=(2 \pi)^{-3 / 2}$. For $A=1$, the factor $(2 \pi)^{3}$ in in eq.(25) should be dropped.
} 
very low energies may correspond to transfer-fission rather than fusion-fission events. This possibility is presently being investigated by these authors [37].

We now consider the fusion cross section for the ${ }^{209} \mathrm{Bi}$ target. In this case the data set [21] does not include energies much below the barrier, as does that of [22]. Fig. 2(b) indicates that the agreement between theory and experiment is much poorer. In this case the results of the optical model calculation without any halo effect (dotted line) are close to the data at above-barrier energies. However, they fall much below the data at energies near and below the barrier. The remaining three calculations, which include halo effects, overestimate the data at above barrier energies. At lower energies the best results are obtained with the optical model calculations with static effects of the halo. The results of our best calculation - the coupled-channel calculations with the Coulomb plus nuclear coupling - are much above the data in the whole energy region.

We should emphasize that our schematic model overestimates the total fusion cross section. Firstly because it cannot include continuum-continuum coupling, which reduces it substantially [13]. Furthermore, the contribution from the breakup channel to the fusion cross section may be overestimated, specially if the projectile breakup is a direct process. In this case, fusion following breakup will be dominantly the incomplete fusion of the ${ }^{4} \mathrm{He}$ fragment with the target. The cross section for incomplete fusion should be much smaller than that for the complete fusion of the ${ }^{6} \mathrm{He}$ projectile. The ${ }^{4} \mathrm{He}$ fragment faces a higher barrier (this is illustrated in Table I) while it carries only a fraction of the collision energy (about $2 / 3$, if the relative energy of the fragments is neglected). Since in our model the effective channel is bound, the contribution from this channel is evaluated as complete fusion and, therefore, is overestimated. It may be a better procedure to neglect the contribution of the effective channel to the total fusion cross section and write $\sigma_{T F} \simeq \sigma_{F}^{(0)}$. Since we do not have reliable information about the breakup mechanism (direct or resonant) our model can only predict that the total fusion cross section should be within the range

$$
\sigma_{F}^{(0)}+\sigma_{F}^{(1)} \gtrsim \sigma_{T F} \gtrsim \sigma_{F}^{(0)}
$$

In Fig. 3 we compare the data with the curves giving the upper and the lower limits indicated in the above equation. The results for the ${ }^{238} \mathrm{U}$ target, shown in figure 3(a), are consistent with the data, except for the lowest data point, where the theoretical prediction is much smaller than the experimental value. In the case of the ${ }^{209} \mathrm{Bi}$ target, the situation is worse. Only the data points at the highest energies fall within the predicted limits. This may be a limitation of our schematic model since it does not contain the continuum-continuum coupling which would substantially reduce the complete fusion cross section [13]. In this way, the dashed line in Fig. 3(b) would be moved down and the agreemente with the data would be better. We should remark that there is an additional discrepancy between theory and the experiment, which occurs for both targets. The experimental slopes of the fusion cross section at low energies are always smaller than the theoretical predictions. In this case, it is unlikely that the discrepancy can be traced back to the limitations of our model. The inclusion of continuumcontinuum coupling does not seem to reduce this slope [13].
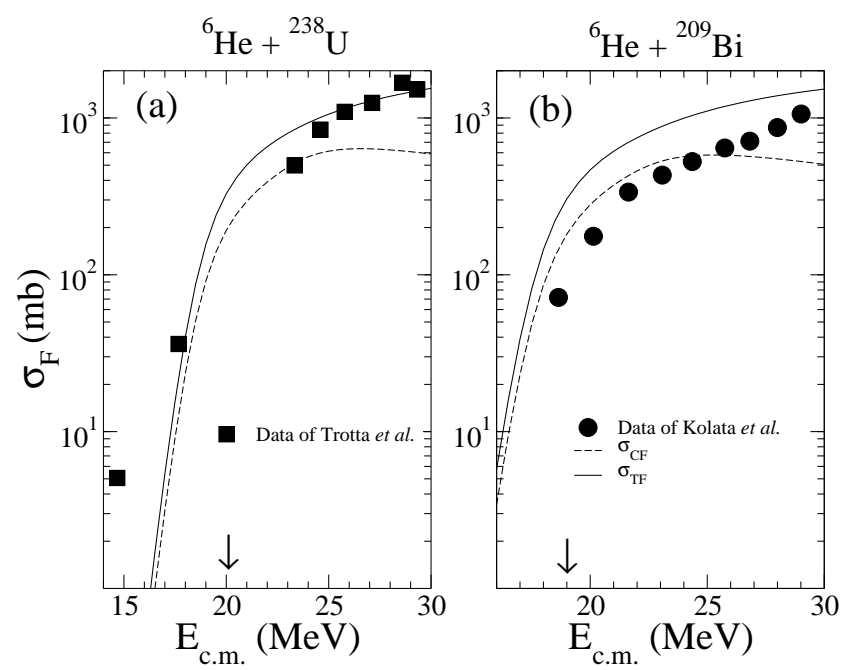

Figure 3. Upper (solid line) and lower (dashed line) limits predicted by our coupled-channel calculations in comparison with the data of [22] (squares) and [21] (circles).

\subsection{Breakup and reaction cross sections}

In Fig. 4 we show angular distributions for the breakup of ${ }^{6} \mathrm{He}$ in collisions with a ${ }^{238} \mathrm{U}$ target. Since the results for the ${ }^{209} \mathrm{Bi}$ target are basically the same, we do not show them in this section. These angular distributions are functions of the deflection angle for the center of mass of the projectile. Usually, this angle must be expressed in terms of the momenta of the fragments. However, the situation is simpler in the present calculation since the breakup channel is treated as a bound state. The calculations were performed for a collision energy about $30 \%$ above the Coulomb barrier. Since there are no data available, we compare results obtained with different approximations.

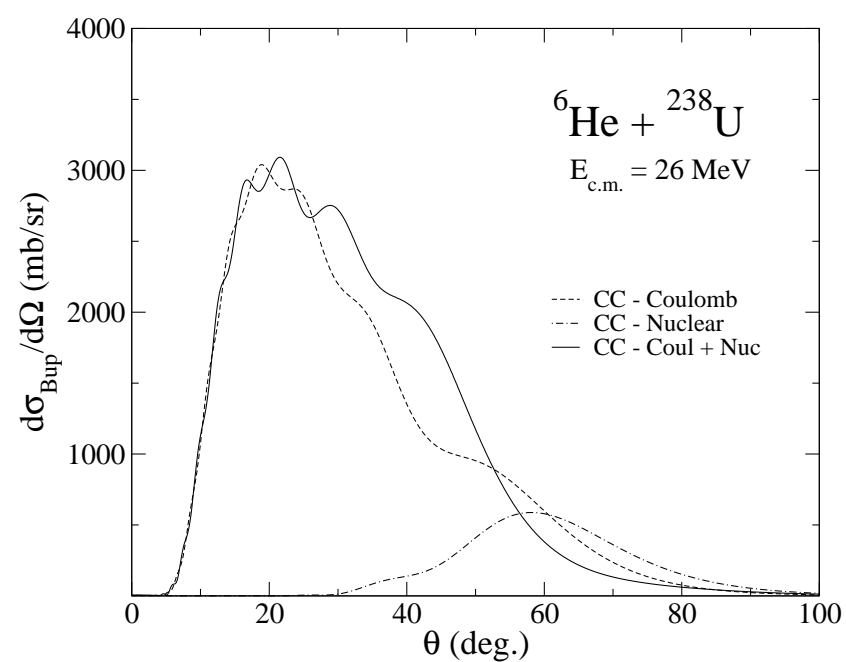

Figure 4. Breakup cross sections in the ${ }^{4} \mathrm{He}+{ }^{238} \mathrm{U}$ collision. The lines are results of coupled-channel calculations with different couplings. For details, see the text. 
The dashed, dot-dashed and solid lines correspond respectively to coupled-channel calculations with Coulomb, nuclear and Coulomb plus nuclear couplings. A comparison of these curves gives relevant information about the roles of these couplings. Firstly, we notice that Coulomb breakup is important over a broad angular region. A second interesting point is that nuclear breakup is negligible at small angles. These angles are associated with distant collisions, for which the short range nuclear form factor is vanishingly small. Nuclear breakup reaches a maximum at the grazing angle $\theta \simeq 60^{\circ}$ and falls off slowly above, owing to fusion absorption. The dashed and the dash-dotted lines cross at $\theta \simeq 60^{\circ}$. Nuclear breakup dominates above this angle and Coulomb breakup dominates below. Comparing the cross sections for each of these processes with that for the coulomb plus nuclear couplings (solid line) one concludes that there is constructive interference below $\sim 50^{\circ}$ and destructive interference above. It is important to remark that Coulomb breakup completeley dominates the angleintegrated cross section. Owing to the factor $(2 l+1)$, appearing in the partial-wave expansion, the main contributions for $\sigma_{B u p}$ come from large partial-waves, where nuclear breakup is very small.

In our two-channel calculations, the reaction cross section is given by

$$
\sigma_{R}=\sigma_{T F}+\sigma_{B u p} .
$$

It can be expanded in partial-waves as

$$
\sigma_{R}=\sum_{l} \sigma_{R}(l)
$$

with

$$
\sigma_{R}(l)=\frac{\pi}{k^{2}}(2 l+1)\left[1-\left|S_{0 l, 0 l}^{l}\right|^{2}\right] .
$$

In Fig. 5 we show $l$ - projected cross sections, $\sigma_{R}(l)$, as functions of $l$. They were obtained with the different approximations discussed in the previous section. The dotted line gives the results of an optical model calculation with the optical potential including the static effects of the halo. In this case there is no coupling with the breakup channel so that the only contribution to $\sigma_{R}$ comes from fusion. For this reason, $\sigma_{R}(l)$ is only relevant at low partial-waves. These waves are associated with close collisions, where the projectile is absorbed by the imaginary potential. In this way, the partial-wave summation of eq.(26) may be truncated at $l_{\text {max }} \simeq 20$. The dot-dashed line are the results of a coupledchannel calculation with only nuclear coupling. Now, the reaction cross section has also contributions from the nuclear breakup. Although the nuclear form factor has a short range, it reaches further than the imaginary potential. The sum in eq.(26) should then include contributions from higher partial-waves, with the truncation value being extented to $l_{\max } \simeq 50$. The dashed line corresponds to a couple-channel calculation with only Coulomb coupling. Now the partialwave expansion converges very slowly. The cut-off angular momentum should be about $l_{\max } \simeq 500$. The solid line was obtained with a coupled-channel calculation with both Coulomb and nuclear couplings. Comparing this curve with those for Coulomb and nuclear coupling separately, we find destructive interference for angular momenta in the range $15 \lesssim l \lesssim 25$ and constructive interference for angular momenta $l \gtrsim 25$. Since large angular momenta are associated with small deflection angles and the other way around, these conclusions are consistent with those reached through Fig. 4.

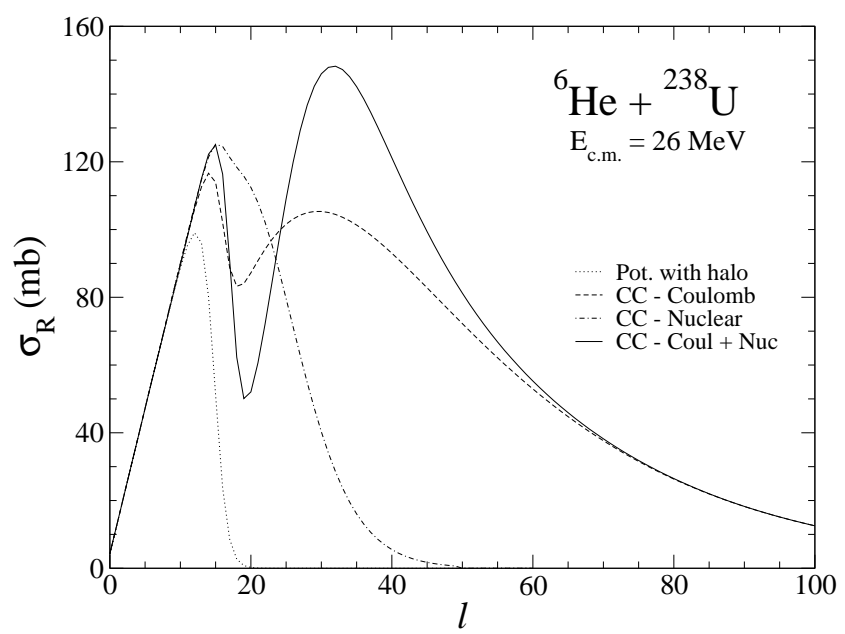

Figure 5. Partial-wave components of the reaction cross section. The dotted line was obtained with an optical model calculation while the remaining lines are results of coupled-channel calculations with different couplings. For details see the text.

We now show, in Fig. 6, the excitation functions obtained by integration of the different contributions shown in Fig. 5. We notice that while the Coulomb breakup is the main contributor to the reaction cross section, there is a cross-over between the fusion and nuclear breakup terms. For the optical model parameters and the nuclear coupling strength employed in this calculation, breakup dominates at energies below $\simeq 32 \mathrm{MeV}$. Two points are worth emphasizing here. One is that for systems for which the Coulomb breakup contribution is small, these two excitation curves indicate the regions where breakup dominates over fusion, or viceversa. Such systems are those for which the dipole contribution to the Coulomb potential is zero, e.g. ${ }^{6} \mathrm{Li} \rightarrow{ }^{4} \mathrm{He}$ $+{ }^{2} \mathrm{H}$. The second point is that the cross-over does not take place at the Coulomb barrier, but a value of the energy which depends on the system, in particular on the binding energy of the fragmenting nucleus, the optical model parameters and the nuclear coupling strength.

\subsection{Elastic Scattering}

In Fig. 7 we present the elastic scattering cross sections calculated with the different approximations discussed in the text. The collision energy is $30 \%$ above the Coulomb barrier and the results are normalized with respect to the Rutherford cross section. Since the cross section for the ${ }^{238} \mathrm{U}$ target turned out to be very similar to that for ${ }^{209} \mathrm{Bi}$, we only show the former. Results of optical model calculations with the optical potential not including static effects of the halo and including them are respectively represented by the dotted and the dashed lines. The dot-dashed line was obtained with a coupled-channel calculation with Coulomb coupling. 
The solid line corresponds to a coupled-channel calculations including both Coulomb and nuclear couplings.

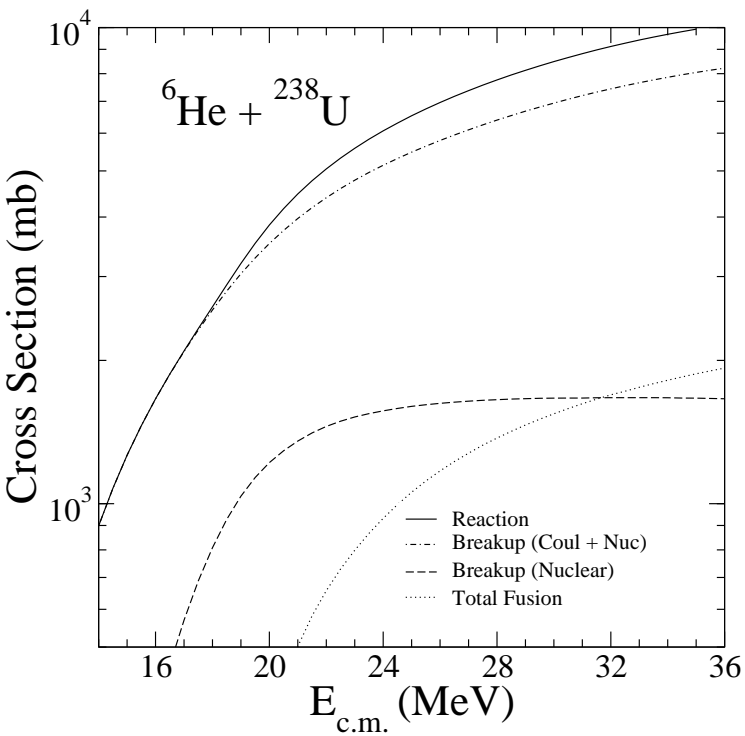

Figure 6. Components of the reaction cross section for the same system and optical model parameters as in Fig. 5.

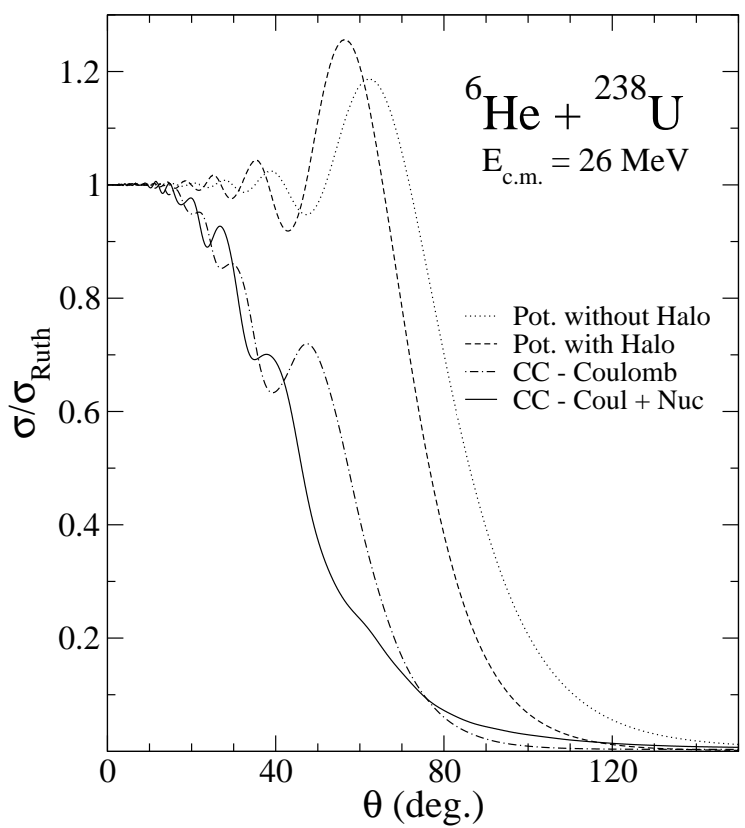

Figure 7. Elastic angular distribution in the ${ }^{6} \mathrm{He}+{ }^{238} \mathrm{U}$ collision. The collision energy is $26 \mathrm{MeV}$ and the results are normalized with respect to the Rutherford cross section. For details, see the text.

The two optical model calculations show some differences. Firstly, the cross section obtained with the halo is smaller at large angles. This results from the reduction of the Coulomb barrier when the halo is taken into account (see table I). A lower Coulomb barrier leads to larger fusion absorption at low partial-waves (large scattering angles) and, thus, to smaller elastic cross sections. The two curves also show differences at smaller angles, between $20^{\circ}$ and the main maximum at $\theta \simeq 60^{\circ}$, where the cross sections oscillate. Since the potential with the halo has a longer range the main maximum is shifted to a smaller angle and the interference between trajectories is slightly intensified.

We now consider the dynamic effects of the halo contained in the coupled-channel calculations. Due to the long range of the electric dipole coupling, the convergence of the calculations requires a very large matching radius $(\sim 360$ $\mathrm{fm})$ and the inclusion of very high partial waves $\left(l_{\max } \sim\right.$ 800 ). We notice that the electric dipole coupling leads to a strong reduction of the elastic cross section, even at small angles. A similar reduction, although less pronounced, is encountered in collisions of deformed nuclei with heavy collision partners [38]. In this case, the loss of flux in the elastic channel arises from Coulomb excitation of rotational states through the electric quadrupole coupling. Comparing the solid and the dot-dashed lines, we conclude that the nuclear coupling leads to further reduction of the cross section over a broad angular region. In spite of its short range, this effect is very important even at angles as low as $50^{\circ}$.

We should mention that elastic scattering measurements of ${ }^{6} \mathrm{He}$ on ${ }^{209} \mathrm{Bi}$ [39] and ${ }^{208} \mathrm{~Pb}$ [40] have recently been reported. The trend of these data follow the one shown in Fig. 7, namely a reduction of the elastic scattering cross section from Rutherford even at small angles. The energies at which these measurement were taken are, respectively $E_{l a b}=22.5 \mathrm{MeV}$ and $29.6 \mathrm{MeV}$ respectively. These energies correspond to $1.2 E_{b}$ and $1.39 E_{b}$ is the barrier height of the system under study. The data in fact show that at, say $\theta_{C M}=25^{\circ}, \sigma / \sigma_{R}=0.6$ in the case of ${ }^{6} \mathrm{He}+\mathrm{Pb}$, to be compared to about unity for the similar, non-exotic system, ${ }^{6} \mathrm{Li}+{ }^{208} \mathrm{~Pb}$. In the case of ${ }^{6} \mathrm{He}+{ }^{209} \mathrm{Bi}$ at the lower of the energies cited above, the corresponding ratio is about 0.9 compared to unity for ${ }^{6} \mathrm{Li}+{ }^{209} \mathrm{Bi}$. It is important to mention that the analyses of the above data were performed with the full CDCC. It is gratifying that our schematic calculation seems to capture the essential physics of the scattering of exotic nuclei.

\section{Conclusions}

We have used the schematic model of ref.[26] to investigate the importance of static and dynamic effects of the ${ }^{6} \mathrm{He}$ halo in collisions with heavy targets. In this model, the two neutrons in the ${ }^{6} \mathrm{He}$ halo are treated as a single particle, the dineutron, and the breakup channel is approximated by a single effective state. Although this model leads to an overestimation of the fusion cross section, it can be used in a qualitative analysis. The coupled-channel calculations used optical potentials derived by the folding method and included Coulomb and nuclear couplings within the dipole approximation.

We have performed calculations of fusion, breakup, reaction and elastic cross sections. In the case of fusion, our results were compared with recent experimental data. Since there are no data available for the remaining cross sections, we have compared results of the different approximations discussed in the text. 
- TABLE I: Potential barriers in the collisions ${ }^{4,6} \mathrm{He}+{ }^{238} \mathrm{U}$ and ${ }^{4,6} \mathrm{He}+{ }^{209} \mathrm{Bi}$. In the case of ${ }^{6} \mathrm{He}$ the table shows the potential barriers for the folding potential with gaussian density (no halo) and with a density that includes a contribution from the halo (with halo).

\begin{tabular}{cccc}
\hline \hline & & & \\
System & ${ }^{4} \mathrm{He}+{ }^{238} \mathrm{U}$ & ${ }^{\mathbf{6}} \mathrm{He}+{ }^{238} \mathrm{U}$ (no halo) & ${ }^{\mathbf{6}} \mathrm{He}+{ }^{238} \mathrm{U}$ (with halo) \\
$V_{B}(\mathrm{MeV})$ & 21.8 & 21.4 & 20.1 \\
& & & \\
System & ${ }^{4} \mathrm{He}+{ }^{209} \mathrm{Bi}$ & ${ }^{\mathbf{6}} \mathrm{He}+{ }^{209} \mathrm{Bi}$ (no halo) & ${ }^{6} \mathrm{He}+{ }^{209} \mathrm{Bi}$ (with halo) \\
$V_{B}(\mathrm{MeV})$ & 21.2 & 20.1 & 19.2 \\
\hline \hline
\end{tabular}

The static effects of the halo on the fusion cross section were shown to be very important above and below the Coulomb barrier. The dynamic effects associated with channel-coupling are more important at sub-barrier energies. Comparing theoretical fusion cross sections with data for the ${ }^{6} \mathrm{He}+{ }^{238} \mathrm{U}$ system, we found that the experimental results are consistent with the upper and lower limits determined in our calculation, except at a very low energy, where the experimental value is much larger that predicted by the theory. In the case of the ${ }^{6} \mathrm{He}+{ }^{209} \mathrm{Bi}$ system, the agreement is worse. The experimental results fall below our lower limit except for energies well above the barrier. We believe that this disagreement is a consequence of the absence of continuum-continuum coupling in our model.

Our calculations of the breakup cross section have shown that Coulomb breakup dominates at angles below $\sim 60^{\circ}$ while the nuclear coupling is more important above this angle. We have also shown that Coulomb-nuclear interference is constructive below $\sim 50^{\circ}$ and destructive above this angle. In the case of elastic scattering, the dynamic effects of the halo were shown to be much more important than the static ones.

The authors are grateful to Prof. H. D. Marta, from the Instituto de Física, Facultad de Ingeniería, Montevideo, Uruguay, for his help in the calculation of the nuclear form factors. We thank Drs. Christian Beck and Monica Trotta for communicating the features of their new experimental results prior to their publication. This work was supported in part by $\mathrm{CNPq}$ and the MCT/FINEP/CNPq(PRONEX) under contract no. 41.96.0886.00. L.F.C. acknowledges partial support from the FAPERJ, and M.S.H. and W.H.Z.C. acknowledge support from the FAPESP.

\section{References}

[1] C.H. Dasso, S. Landowne, and A. Winther, Nucl. Phys. A 432, 495 (1985).

[2] S.G. Steadman, ed., "Fusion Reactions Below the Coulomb Barrier", Springer Verlag, Berlin Heidelberg New York Tokyo, 1984.

[3] M. Beckerman, Rep. Prog. Phys. 51, 281 (1993).

[4] C.A. Bertulani, L.F. Canto, and M.S. Hussein, Phys. Rep. 226, 281 (1993); C.A. Bertulani, M.S. Hussein, and G. Münzenberg, "Physics of Radioactive Beams" (Nova Science, New York, 2001); M. S. Hussein, L.F. Canto, and R. Donangelo, Nucl. Phys. A722, 321c (2003).
[5] N. Takigawa and H. Sagawa, Phys. Letters B265, 23 (1991).

[6] M.S. Hussein, M.P. Pato, L.F. Canto, and R. Donangelo, Phys. Rev. C46, 377 (1992); C47, 2398 (1993).

[7] N. Takigawa, M. Kuratani, and H. Sagawa, Phys. Rev. C47 R2470 (1993).

[8] C.H. Dasso and A. Vitturi, Phys. Rev. C50, R12 (1994).

[9] L.F. Canto, R. Donangelo, Lia M. Matos, M.S. Hussein, and P. Lotti, Phys. Rev. C58, 1107 (1998).

[10] M. Dasgupta et al., Phys. Rev. C66, 041602R (2002).

[11] L.F. Canto, R. Donangelo, M.S. Hussein, and M.P. Pato, Nucl. Phys. A542, 131 (1992).

[12] K. Hagino, A. Vitturi, C.H. Dasso, and S. Lenzi, Phys. Rev. C61, 037602 (2000).

[13] A. Diaz-Torres and I.J. Thompson, Phys. Rev. C65, 024606 (2002).

[14] A. Diaz-Torres, I.J. Thompson, and C. Beck, Phys. Rev. C68, 044607 (2003).

[15] I.J. Thompson, Comput. Phys. Rep. 7, 167 (1988).

[16] Y. Sakuragi, M. Yashiro, and M. Kamimura, Prog. Theo. Phys. Suppl. 89, 136 (1986).

[17] V. Fekou-Youmbi et al., Nucl. Phys. A538, 811 (1995).

[18] A. Yoshida et al., Phys. Letters B389, 457 (1996).

[19] C. Signorini et al., Eur. Phys. J. A2, 227 (1998).

[20] C. Signorini et al., Eur. Phys. J. A13, 129 (2000).

[21] J.J. Kolata et. al., Phys. Rev. Lett. 81, 4580 (1998).

[22] M. Trotta et al.,Phys. Rev. Lett. 84, 11 (2000).

[23] N. Alamanos, et al., Phys. Rev. C65, 054606 (2002).

[24] K.E. Rehm et. al., Phys. Rev. Lett. 81, 3341 (1998).

[25] K.E. Zyromski et al., Phys. Rev. C55, R562 (1997).

[26] W.H.Z. Cárdenas, L.F. Canto, R. Donangelo, H.S. Hussein, and N. Carlin Filho, Phys. Rev. C68, 054614 (2003).

[27] G.R. Satchler, "Direct Nuclear Reactions", Oxford University Press, 1983

[28] D.G. Madland and P.G. Young, Los Alamos Report No. LA7533-mb (1978) (unpublished).

[29] G.D. Alkhazov et al. Phys. Rev. Lett. 782313 (1997).

[30] Yu. N. Eldyshev, V. N. Lukyanov, and Yu. S. Pol., Sov. J. Nucl. Phys. 16, 282 (1973).

[31] A. Romanelli, L.F. Canto, R. Donangelo, and P. Lotti, Nucl. Phys. A588, 71c (1995); H. Esbensen, G.F. Bertsch, and C.A. Bertulani, Nucl. Phys. A581, 107 (1995); L.F. Canto, R. Donangelo, and A. Romanelli, Phys. Rev. C53, 3147 (1996). 
[32] A.M.S. Breitschaft et al., Ann. of Phys. 243, 420 (1995).

[33] A.R. Edmonds, "Angular Momentum in Quantum Mechanics", Princeton University Press, Princeton, New Jersey, 1974.

[34] V.E. Viola and T. Sikkeland, Phys. Rev. 128, 767 (1962).

[35] W.J. Ramler et al. Phys. Rev. 114, 154 (1959).

[36] A.R. Barnett and J.L. Lilley, Phys. Rev. C9, 2010 (1974).

[37] M. Trotta, private communication.
[38] W.G. Love, T. Terasawa, and G.R. Satchler, Phys. Rev. Letters 39, 6 (1977).

[39] N. Keeley, J. M. Cook, K. W. Kemper, B. T. Roeder, W. D. Weintrap, F. Marechal, and K. Rusek, Phys. Rev. C68, 054601 (2003).

[40] K. Rusek, N. Keeley, K. W. Kemper, and R. Raabe, Phys. Rev. C 67, 041604 (2003). 